\title{
Zearalenone induces alterations of hepatic immune responses by modulation of pro-inflammatory cytokines and matrix metalloproteinase gene expression
}

\author{
G. Pistol, M. Gras, D. E. Marin, C. Tabuc and I. Taranu \\ Laboratory of Animal Biology, INCDBNA, National Research-Development Institute of Biology and Animal Nutrition, \\ Balotesti, Romania
}

\begin{abstract}
Zearalenone (ZEA) is an estrogenic mycotoxin produced by Fusarium graminearum and by other Fusarium species. ZEA is commonly found as contaminant of stored grain and has chronic estrogenic effects on mammals, being a risk factor from both public health and agricultural perspectives. Also, ZEA and its derivatives may have divergent effects on important parameters of swine innate immunity and are described as modulators of pro-inflammatory cytokine expression. For zearalenone and other Fusarium contaminants (ochratoxin, deoxynivalenol, fumonisin B1+B2) the tolerance limits for pig are regulated only by recommendation CE/576/2006, which means that new experimental evidences (in-depth investigation at the cellular level) are required in order to confirm the proposed/recommended limit and record it as limit. The elaboration of a regulation and its enactment is imperiously necessary.

Starting from these evidences, a feeding trial was conducted to evaluate the effect of a ZEA-contaminated diet on immune response in young pigs. Emerging evidence suggests that the liver is an important part of the body's immune response. The effect of ZEA on hepatic pro-inflammatory cytokines (TNF $\alpha$, IL-8, IL-6, IL-1 $\beta$, and IFN $\gamma$ ) and other molecules involved in inflammatory processes (MMPs, TIMPs, PPAR $\gamma$ ) in weaned pigs was investigated in this in vivo study.

A contaminated diet, containing zearalenone $(250 \mathrm{ppb})$ was given to 5 weaned piglets for a period of 18 days. A group of 5 piglets which received uncontaminated diet was used as control group. In order to evaluate effects of ZEA on several hepatic immune responses, we perform real-time PCR analysis for MMPs, TIMPs and pro-inflammatory cytokines gene expression using cDNA obtained from liver samples.

Our results showed that a concentration of around $250 \mathrm{ppb}$ ZEA lead to a statistically significant marked decrease of hepatic MMPs gene expression (MMP-2: 0.19, and MMP-9: 0.02 fold change), correlated with an decrease in TIMPs (TIMP-1: 0.19; TIMP-2: 0.21 fold change) mRNA. Concomitant with an increase in PPAR $\gamma$ mRNA (1.24 fold change), at hepatic level, consumption of dietary ZEA induces dramatically reduction of pro-inflammatory cytokines gene expression; this downregulation of mRNA was observed for all investigated pro-inflammatory cytokines (TNF $\alpha$ : 0.16; IL-8: 0.36; IL-6: 0.09; IL-1 $\beta$ : 0.15 and IFN $\gamma: 0.11$ fold change, respectively) in the liver of experimentally intoxicated animals.

In conclusion, our study shows that ZEA altered several parameters of the hepatic cellular immune response. Therefore, our results represent additional data that could be taken into account in the determination of the regulation limit of the tolerance to ZEA. Also, supplementary in-depth studies must be performed in order to determine effects of ZEA at cellular and molecular level.
\end{abstract}

